\title{
Errance diagnostique devant un hématome spontané récidivant du menton
}

\author{
Guilloud $\mathrm{C}^{\mathbf{1}}$, Boisramé-Gastrin $\mathrm{S}^{\mathbf{1}}$, Chastaing $\mathbf{M}^{\mathbf{2}}$, Prédine-Hug $\mathrm{F}^{\mathbf{1}}$, Berthou $\mathrm{C}^{\mathbf{3}}$ \\ ${ }^{1}$ UFR d'Odontologie, Service d'Odontologie, CHU, Brest, France \\ ${ }^{2}$ Service de Psychologie médicale et de Psychiatrie de Liaison, CHU, Brest, France \\ ${ }^{3}$ Service d'Hématologie, CHU, Brest, France \\ christelle.guilloud@unige.ch
}

Un hématome sous-cutané d'apparition brutale et spontanée nécessite une démarche diagnostique rigoureuse. En effet, il peut être révélateur de pathologies diverses, bénignes ou malignes, comme un trouble de la coagulation, inné ou acquis, une pathologie infectieuse, voire une affection tumorale.

Nous rapportons la prise en charge d'un cas d'hématome spontané, récidivant de la région mentonnière. Une jeune femme de 15 ans, est adressée par le Service d'Hématologie pour un hématome spontané mentonnier, nécessitant une hospitalisation pour une douleur estimée à 8 sur l'échelle visuelle analogique. Dans les antécédents médico-chirurgicaux, on retrouve une leucémie lymphoblastique aiguë, traitée par chimiothérapie à l'âge de 4 ans. Les différents examens complémentaires (bilan sanguin, sérologies virales, CT-scan, IRM) ne mettent en évidence rien de particulier. La lésion disparait en 1 semaine. Ses parents rapportent un second épisode d'hématome spontané 2 mois plus tard, de même localisation, sans prise en charge hospitalière. Lors d'une consultation de contrôle, la mère signale une aménorrhée de plus de quatre mois chez sa fille. Devant l'hypothèse d'une maladie de Cushing, elle est adressée dans le Service d'Endocrinologie qui, après explorations, ne retrouve aucune anomalie. Trois jours après cette consultation, ses parents l'adressent en urgence pour un nouvel hématome intéressant le sillon nasogénien. A ce tableau clinique, sont associés une dysphagie et une douleur de palier III. Une échographie de la zone permet d'écarter une malformation artério-veineuse ou un lymphangiome kystique. Les résultats des bilans effectués sont normaux. L'analyse anatomo-pathologique de la biopsie de la face interne de la lèvre inférieure révèle des petits foyers d'inflammation chronique périvasculaire sans caractères spécifiques.

Aucun diagnostic n'a encore été posé pour cette jeune fille qui a présenté, en 6 mois, 3 épisodes d'hématome facial spontané, ayant tendance à diffuser. Devant l'absence d'étiologie et à sa demande, la jeune fille, de nature anxio-dépressive, est adressée à la consultation psychiatrique de l'hôpital (Uthman et al. 2000, Boussault et al. 2005) 